\title{
Are gastroenterologists willing to implement imaging- guided surveillance for Barrett's esophagus? Results from a national survey
}

Authors

Institutions
Anoop Appannagari ${ }^{1}$, A. Samad Soudagar ${ }^{1}$, Constance Pietrzak ${ }^{1}$, Prateek Sharma ${ }^{2}$, Neil Gupta ${ }^{1}$

${ }^{1}$ Loyola University Medical Center, Department of Gastroenterology, Maywood, Illinois, United States

${ }^{2}$ University of Kansas Medical Center, Department of Gastroenterology, Kansas City, Kansas, United States submitted

31. December 2014 accepted after revision

7. January 2015

\section{Bibliography}

DOI http://dx.doi.org/

$10.1055 / \mathrm{s}-0034-1391413$

Published online: 6.5.2015

Endosc Int Open 2015; 03:

E181-E185

(c) Georg Thieme Verlag KG

Stuttgart · New York

E-ISSN 2196-9736

Corresponding author

\section{Neil Gupta}

2160 S. 1st Avenue, Building 54, Room 167

Maywood, Illinois 60153

United States

Fax: 1-708-216-4113

negupta@lumc.edu
Introduction: The American Society for Gastrointestinal Endoscopy (ASGE) has published a Preservation and Incorporation of Valuable Endoscopic Innovations (PIVI) statement on incorporating an imaging-guided surveillance protocol to replace the current practice of four-quadrant biopsies every two centimeters for Barrett's esophagus (BE) surveillance. We sought to determine if current gastroenterologists would be willing to apply these changes to their practice and identify any barriers to implementation.

Methods: We collected data using surveys that were distributed at two national meetings and using a random selection process emailed surveys to members listed in the American Gastroenterological Association directory. Physicians from a variety of practice settings participated. Primary outcomes of our study included determining whether clinicians would be willing to accept an imaging-based surveillance protocol, their reasons for not doing so, and whether a financial incentive would be persuade them to implement the protocol. Continuous variables were reported as mean \pm standard deviation. Categori-

\section{Abbreviations \\ $\nabla$ \\ ASGE American Society for Gastrointestinal Endoscopy \\ BE Barrett's esophagus \\ EUS endoscopic ultrasound \\ PIVI Preservation and Incorporation of Valuable Endoscopic Innovations}

\section{Background \\ $\nabla$}

The American Society for Gastrointestinal Endoscopy (ASGE) recently published a Preservation and Incorporation of Valuable Endoscopic Innovations (PIVI) statement on using an imaging-guided surveillance protocol rather than the current cal variables were summarized with percentages and $95 \%$ confidence intervals.

Results: Gastroenterologists (172) completed the survey; and 140 (81.4\%) of them stated they would implemented the PIVI recommendations into practice. Using a multivariate analysis of the data, physicians who reported a financial incentive for submitting biopsy specimens to pathology were less likely to implement the PIVI recommendations. The two main barriers to implementation of the protocol were medical-legal and financial reasons. Of the 32 gastroenterologists who were not willing to implement the imagingguided surveillance protocol, 20 (62.5\%) stated that they would implement it if there were a financial incentive.

Discussion: The PIVI statement focuses on reevaluating our current method of surveillance for BE. The results of our survey show that gastroenterologists may be willing to implement an imaging-guided surveillance program, but concerns regarding financial compensation and proper training in advanced imaging techniques remain.

protocol of obtaining four-quadrant biopsies every two centimeters for diagnosis of Barrett's esophagus (BE) [1]. The PIVI statement was created to address important clinical questions and other issues related to endoscopic innovation. Endoscopic surveillance of BE falls into this category because the current protocol has many limitations and weaknesses. Distinguishing dysplasia and adenocarcinoma from non-dysplastic tissue can be difficult using standard white-light endoscopy. Moreover, it is difficult for clinicians to perform four-quadrant biopsies every two centimeters [2]. Abrams et al found that adherence to this regimen is accomplished in only $51 \%$ of patients and it is even more difficult with longer segments of BE [3]. Thus, the PIVI committee proposed a system utilizing advanced imaging tech- 
niques to create a more targeted protocol for performing biopsies to reduce the number of patients biopsied for non-dysplastic disease.

With the implementation of any new technology, an important factor to consider is the willingness of gastroenterologists to utilize and accept these technologies. Therefore, we aimed to determine whether gastrointestinal physicians would be willing to implement imaging-guided surveillance for BE and to identify potential barriers to implementation of this protocol in clinical practice.

\section{Materials and methods}

$\nabla$

\section{Survey method}

Our data was collected using surveys that were distributed in two ways. First, we attended both Digestive Disease Week (DDW) 2013 and American College of Gastroenterology (ACG) 2013 meetings, two large, national meetings of gastroenterologists, and randomly distributed the survey to meeting participants. Second, we randomly contacted clinicians by email using addresses obtained from the American Gastroenterological Association directory to distribute the survey. The survey consisted of 14 questions that included yes/no questions, free response questions, and multiple choice questions. The survey was divided into two sections that included demographics and questions specifically related to the PIVI statement. If the clinicians were willing to participate, they responded by writing answers on the questionnaire ( $\bullet$ Fig. 1).

\section{Questionnaire}

Demographic data were collected, including age, number of years in practice, clinical setting, size of the clinician's respective cities, board certification status in both gastroenterology and transplant hepatology, number of esophagogastroduodenoscopies performed for BE surveillance, their practice for specimen submission to pathology and whether a financial incentive was received for doing so, and their experience with advanced imaging. Regarding the PIVI statement, we specifically inquired about the clinician's awareness of the PIVI statement, their willingness to implement advanced imaging strategies, and their reasons for not implementing advanced imaging strategies into practice. The reasons for not doing implementing advanced imaging strategies that we proposed included the effort required for learning and/or using new imaging technologies, the lack of availability of new imaging technologies, the cost, the financial disincentive that arises in not sending BE biopsies to pathology, the medicolegal concerns, and other. Participants were allowed to choose multiple reasons. The questionnaire asked if clinicians would be willing to use new imaging technologies if a financial incentive was provided. If those clinicians answered "yes" or "more likely to do so," we asked them to specify dollar amounts; the choices were $<\$ 50, \$ 51-\$ 100, \$ 101-\$ 150, \$ 150-\$ 200$, and $>\$ 201$. We did not include any gastroenterology trainees as participants in the study. Primary outcomes of the study included the participant's willingness to accept an imaging-based surveillance protocol for BE, their reasons for not doing so, and whether a financial incentive would persuade them to implement an imaging-based surveillance protocol. The participant's responses to the questionnaire were entered into a software database (Microsoft Excel; Microsoft Corporation., Redmond, Washington, United States).

\section{Statistical analysis}

Continuous variables were reported as mean \pm standard deviation and categorical variables were summarized with percentages and $95 \%$ confidence intervals. Multivariate regression analyses were used to identify independent predictors for the following outcomes: awareness of the ASGE PIVI statement, willingness to implement imaging-guided surveillance of $B E$, and the amount of financial compensation perceived to be appropriate for performing imaging-guided surveillance of BE. Physician age, years in practice, practice setting (academic vs. private practice), city size, board certification in gastroenterology, board certification in transplant hepatology, current performance of endoscopic retrograde cholangiopancreatography and/or endoscopic ultrasound, current performance of clinical or basic science research, average number of esophagogastroduodenoscopies performed for BE surveillance per month, financial incentive for submitting biopsy specimens to pathology, and current use of advanced imaging technologies during esophagogastroduodenoscopy were included as potential predictor variables. A $P<0.05$ was required for statistical significance. Analyses were performed using STATA/IC version 10.1 (StataCorp; College Station, Texas).

\section{Results \\ $\nabla$}

\section{Participant details}

Gastroenterologists (172) completed the survey: 145 participants were contacted at national meetings ( $100 \%$ response rate) and 27 participants were contacted by email (3.9\% response rate). - Table 1 displays the characteristics of those who participated in the survey. The mean (SD) age of the participants was 46.6 (9.7) years and time in practice was 11.8 (9.3) years. Sixty-six respondents (38.4\%) practiced in an academic setting while the others were in private practice. $28(16.3 \%)$ reported having a financial incentive for submitting biopsy specimens to pathology such as having ownership in a pathology facility, and 94 (54.6\%) reported that they currently used some form of advanced imaging (chromoendoscopy, electronic chromoendoscopy, confocal laser endomicroscopy, etc.) in their upper gastrointestinal endoscopy practice.

\section{Awareness and implementation of imaging-guided surveillance of Barrett's esophagus}

Of the 172 gastroenterologists who completed the survey, 95 (55.2\%) of them stated they were aware of the ASGE PIVI statement proposing imaging-guided surveillance of BE. One-hundred forty $(81.4 \%)$ of them stated they were willing to implement imaging-guided surveillance if an imaging technology met the PIVI threshold and if adequate training in such technology were available. Multivariate analysis of the data showed that participants who were board-certified in transplant hepatology were less likely to be aware of the ASGE PIVI statement on imagingguided surveillance of BE (OR 0.26, 95\%CI $0.07-0.88$ ). In addition, physicians reporting a financial incentive for submitting biopsy specimens to pathology were less likely to be willing to implement the imaging-guided surveillance of $\mathrm{BE}$ (OR, 0.17; $95 \% \mathrm{CI} 0.06-0.48$ ). These were the only two independent predictors for awareness and willingness to implement imagingguided surveillance of $\mathrm{BE}$. 
Thank you for participating in our survey about real-time imaging of Barrett's esophagus.

1. What is your age? years

2. Are you currently board certified in gastroenterology?

- Yes

- No

3. Are you currently board certified in transplant hepatology?

_Yes

- No

4. Do you currently conduct clinical or basic science research?

- Yes

- No

5. Do you perform ERCP or EUS?

- Yes

- No

6. How long have you been a practicing gastroenterologist (in years)?

7. In what setting do you primarily practice?

_ Academics/University practice

_ Hospital employed private practice

_ Non-hospital employed private practice

8. In what size city do you currently practice (in terms of population size)?

- $<25,000$

25,000-250,000

_ $>250,000-1,000,000,000$

- $>1,000,000$

9. On average, how many EGD for Barrett's surveillance do you perform in a month?

- None

$-0-5$

$-6-10$

$-11-15$

- $>16$

10. Which of the following imaging techniques do you currently use while performing EGD in your practice? (check all that apply)

_Standard white light endoscopy (not high definition)

_ High definition white light endoscopy

_Electronic chromoendoscopy (Narrow band imaging, I-scan, FICE, etc)

_ Chemical chromoendoscopy (methylene blue, acetic acid, indigo

carmine, etc)

_Confocal laser endomicroscopy

Fig. 1 Reproduction of questionnaire used in this study.
11. Do you currently have a financial incentive for submitting biopsy specimens for histopathology?

- Yes

- No

The ASGE recently published a PIVI (Preservation and Incorporation of Valuable Endoscopic Innovations) statement for imaging guided surveillance in Barrett's esophagus patients. They concluded that an endoscopic imaging-based surveillance test must have a sensitivity and specificity of at least $90 \%$ and $80 \%$, respectively, in order to replace the current biopsybased surveillance protocol.

12. Are you aware of this PIVI statement?

-Yes

_No

13. If new imaging technologies existed that met the PIVI performance thresholds and you were provided appropriate training, would you be willing to adopt this practice for Barrett's surveillance?

- Yes

- No

a. If no, what would be your reason for not doing so? (choose as many as apply)

_ Effort required for learning and/or using new imaging technologies

_ Lack of availability of new imaging technologies

_ Cost of new imaging technologies

_ Financial disincentive in not sending Barrett's biopsies to pathology

- Medico-legal concerns regarding assigning incorrect grade of dysplasia without tissue biopsy

_ Other (please specify)

14. If financial incentive/reimbursement were provided to perform realtime advance imaging endoscopic surveillance without using the current standard 4-quadrant biopsy every $2 \mathrm{~cm}$ protocol, and this service was available in your practice, would you be willing to use new imaging technologies?

_Yes

_ No

_More likely to do so

a. If "yes" or "more likely to do so," how much financial incentive per patient would be appropriate?

- $>\$ 201$

- $\$ 150-200$

$\$ 101-150$

$\$ 51-100$

$-<\$ 50$

\section{Barriers to implementation}

Of the 32 gastroenterologists who were unwilling to implement the imaging-guided surveillance of $\mathrm{BE}, 28$ of them listed medicallegal concerns and 12 of them listed financial disincentives and/ or the lack of financial incentives as barriers to implementing imaging-guided surveillance of BE. Twenty of these 32 gastroenterologists $(62.5 \%)$ stated they would be willing to implement imaging-guided surveillance of $\mathrm{BE}$ if given a financial incentive to do so. Only 143 gastroenterologists ( $83 \%$ ) who completed the survey provided information on how much financial incentive would be appropriate for performing an in vivo optical diagnosis of $\mathrm{BE}$ histology. Of these 143 gastroenterologists, the response was as follows: $14(9.8 \%)<\$ 50,14(9.8 \%) \$ 51-\$ 100,15(10.5 \%)$
$\$ 101-\$ 150,24(16.8 \%) \$ 151$ - \$200, and $76(53.1 \%)>\$ 201$. Multivariate analysis of the data showed that physicians in academic practice $(\mathrm{OR}, 0.10$; $95 \% \mathrm{CI} 0.02-0.41)$ and those with longer durations in practice (OR, $0.84 ; 95 \% \mathrm{CI} 0.72-0.99)$ were less likely to feel that $\$ 200$ or more was appropriate compensation for providing an in vivo optical diagnosis of BE histology.

\section{Discussion}

$\nabla$

This descriptive, cross-sectional observational study set out to determine the willingness of current gastrointestinal physicians to implement an imaging-based surveillance protocol for BE. Re- 


\begin{tabular}{|c|c|c|c|}
\hline & $\begin{array}{l}\text { Academic practice } \\
(n=66)\end{array}$ & $\begin{array}{l}\text { Private practice } \\
(n=106)\end{array}$ & $P$-value \\
\hline \multicolumn{4}{|l|}{ Demographics } \\
\hline Age, mean (SD), years & $44.0(9.5)$ & $48.2(9.5)$ & NS \\
\hline Length of practice, mean (SD) years & $9.5(9.0)$ & $13.3(9.3)$ & NS \\
\hline City population >1,000,000, average (\%) & $37(56 \%)$ & $41(39)$ & 0.05 \\
\hline \multicolumn{4}{|c|}{ Non-imaging diagnostics performed/week, average (\%) } \\
\hline $0-5$ & $18(27)$ & $9(8)$ & \\
\hline $6-10$ & $33(50)$ & $40(38)$ & $<0.001$ \\
\hline $11-15$ & $12(18)$ & $34(32)$ & \\
\hline$>15$ & $3(5)$ & $23(22$ & \\
\hline \multicolumn{4}{|l|}{ Respondents, number (\%) } \\
\hline \multicolumn{4}{|l|}{ With access to } \\
\hline Confocal laser endomicroscopy & $30(45)$ & $16(15)$ & $<0.001$ \\
\hline Electronic chromoendoscopy & $45(68)$ & $44(42)$ & 0.001 \\
\hline Chemical chromoendoscopy & $26(39)$ & $7(7)$ & $<0.001$ \\
\hline High definition white light & $59(89)$ & $97(92)$ & NS \\
\hline Aware of PIVI statement & $49(74)$ & $46(43)$ & $<0.001$ \\
\hline With financial incentives to use pathology lab & $0(0)$ & $28(26)$ & $<0.001$ \\
\hline Willing to use imaging-guided BE surveillance & $64(97)$ & $76(72)$ & $<0.001$ \\
\hline With financial incentive & $54(82)$ & $93(88)$ & NS \\
\hline \multicolumn{4}{|l|}{ Preferred financial incentive } \\
\hline$>\$ 200$ & $16(31)$ & $60(67)$ & \\
\hline$\$ 100-\$ 200$ & $15(29)$ & $23(25)$ & \\
\hline$<\$ 100$ & $21(40)$ & $7(8)$ & \\
\hline
\end{tabular}

Table 1 Characteristics of academic and private practice gastroenterologists surveyed.

Abbreviations: SD, standard deviation; NS, not statistically significant; PIVI, Preservation and Incorporation of Valuable Endoscopic Innovations; BE, Barrett's esophagus.

sult of surveys show that over $80 \%$ of gastroenterologists would be willing to implement an imaging-based surveillance protocol into their practice. Given the many problems with our current BE surveillance regimen, this result is not surprising and is encouraging. Advanced imaging techniques have been shown to be a superior to other methods in detecting cancer or dysplasia [4]. Quemseya et al. performed a meta-analysis of 14 relevant studies and found that advanced imaging techniques increased diagnosis of dysplasia/cancer by 34\% [5]. Subgroup analysis showed an increase in both advanced imaging techniques analyzed (chromoendoscopy and virtual chromoendoscopy). Another study by Sharma et al. examined probe-based confocal laser endomicroscopy and found that when combined with high-definition white-light endoscopy the ability to detect neoplasia was improved [6]. No technologies currently available meet all of the PIVI thresholds. It is important to remember that high-quality endoscopic examination is important in implementing any imaging-based protocol.

Gastroenterologists surveyed gave medical-legal concerns as their primary reasons for not implementing an imaging-based $\mathrm{BE}$ surveillance protocol. It is possible that given the lack of adequate training in reading and applying advanced imaging techniques, gastroenterologists are concerned with the theoretical risk of misreading the imaging and potentially missing the diagnosis of a patient with high-grade dysplasia or cancer. Training programs would have to be implemented to instruct gastroenterologists in the imaging methods that meet the PIVI requirements. Training could involve video modules, shadowing experienced gastroenterologists, and hands-on training using both models and actual patients. Additional studies are needed to quantify how much training would be needed before a physician is deemed competent to apply these modalities in clinical practice. Guidelines specific to each type of advanced imaging technique, including optical electronic enhanced endoscopy, high resolution endoscopy with magnification, and dye-based image enhanced endoscopy, would be needed. The kinds of documentation needed for pathology and imaging-guided surveillance are different. In the current system, pathologists review the biopsies and create a report, however, when using imaging-guided surveillance, the endoscopist will need to save high resolution images for documentation. Quality control is also a concern because pathologists currently have the ability to review slides with fellow pathologists for confirmation of findings. There will need to be a quality control mechanism for imaging-guided surveillance.

We found that $40 \%$ of gastroenterologists stated that they would not implement an imaging-guided BE surveillance system into practice due to the lack of a financial incentive. In their current practices, physicians can bill for biopsies taken during BE surveillance, whereas there is no payment structure for "optical" biopsies obtained using advanced imaging techniques. Moreover, many gastroenterologists receive compensation for regular biopsies because they have a stake in the ownership of their pathology laboratories. If they implement imaging-guided BE surveillance, they would lose that source of revenue.

A regular biopsy and an optical biopsy share the same goal: to evaluate tissue for intestinal metaplasia, dysplasia, and malignancy. Multivariate analysis showed that physicians who currently receive a financial incentive for submitting tissues to pathology for analysis were less likely to be willing to implement imagingguided BE surveillance. In addition, considerable time and effort are needed for the gastroenterologist to learn a new method; time that takes them away from clinical duties for which they receive compensation. Further, clinicians would be taking the responsibility and the liability of now born by pathologists, which is another reason many gastroenterologists feel there would be a need for additional financial compensation. Over one-half of the physicians who responded to the survey stated that they would want $\$ 201$ (the highest category given on the survey) for imaging-guided surveillance of BE. As expected, physicians at academic medical centers were less likely to feel that $\$ 201$ or more 
was appropriate compensation for providing an imaging-guided diagnosis because compensation models differ between academic and private practices. The reimbursement for submitting a single sample to pathology (about $\$ 70.00$; that is, $\$ 38.00$ for the physician and $\$ 32.00$ for the laboratory fee) [7], it seems reasonable that clinicians would expect some level of compensation. Long-segment BE typically requires multiple samples be sent to pathology, an even stronger case can be made for providing compensation to gastroenterologists for their use of imaging-guided BE surveillance. Obviously, introducing any financial compensation will reduce the overall cost-effectiveness of an imagingguided BE surveillance protocol. The advantages of the imagingguided protocol must be weighed against reducing pathology costs and improving diagnostic accuracy. Future studies will be needed when a financial compensation model is in place for the gastroenterologists who use an imaging-guided BE surveillance protocol.

Limitations of this study include a limited sample size, the low response to emails, and the accuracy of survey answers, which can be an issue with any survey administered. Physicians at national meetings are often busy and surveys may have been filled out in haste with less than accurate answers provided. Physicians surveyed were also all gastroenterologists from the United States. Their views and opinions may differ from gastroenterologists around the world, and any comments on financial incentives would not necessarily apply to the international population of gastroenterologists. Our particular sample of gastroenterologists was over-represented by academic gastroenterologists with access to advanced imaging techniques. This may have imposed a sampling bias, however, one can also argue that these clinicians have the most experience using advanced imaging techniques and would be intimately aware of their drawbacks. In addition, the financial incentives may have been under-reported because physicians may not have felt comfortable disclosing that information.
The PIVI statement highlights the importance of re-thinking the current practice of surveillance for BE. Using a survey of gastroenterologists in a variety of practice settings, we found that $80 \%$ of them were willing to adopt an imaging-guided surveillance strategy for BE. Concerns among gastroenterologists include medical-legal issues and financial compensation. Strategies to address these concerns will be needed before imaging-guided BE surveillance is widespread.

Competing interests: Neil Gupta has received research grants from Cook Medical, CDx Diagnostics and Cosmo Pharmaceuticals. Prateek Sharma receives grant support from Effexus, CDx Diagnostics, Olympus Inc., and Nine Point.

\section{References}

1 Sharma P, Savides TJ, Canto MI et al. The American Society for Gastrointestinal Endoscopy PIVI (Preservation and Incorporation of Valuable Endoscopic Innovations) on imaging in Barrett's esophagus. Gastrointest Endosc 2012; 76: 252-254

2 Canto MI, Setrakian S, Willis J et al. Methylene blue-directed biopsies improve detection of intestinal metaplasia and dysplasia in Barrett's esophagus. Gastrointest Endosc 2000; 51: 560 - 568

3 Abrams JA, Kapel RC, Lindberg GM et al. Adherence to biopsy guidelines for Barrett's esophagus surveillance in the community setting in the United States. Clin Gastroenterol Hepatol 2009; 7: 736 - 742

4 Goda K, Tajiri H, Ikegami $M$ et al. Usefulness of magnifying endoscopy with narrow band imaging for the detection of specialized intestinal metaplasia in columnar-lined esophagus and Barrett's adenocarcinoma. Gastrointest Endosc 2007; 65: 36-46

5 Qumseya BJ, Wang H, Badie $N$ et al. Advanced imaging technologies increase detection of dysplasia and neoplasia in patients with Barrett's esophagus: a meta-analysis and systematic review. Clin Gastroenterol Hepatol 2013; 11: $1562-1570$

6 Sharma P, Meining AR, Coron E et al. Real-time increased detection of neoplastic tissue in Barrett's esophagus with probe-based confocal laser endomicroscopy: final results of an international multicenter, prospective, randomized, controlled trial. Gastrointest Endosc 2011; 74: $465-472$

7 Centers for Medicare \& Medicaid Services. (Internet). Baltimore (MD): Available from www.cms.gov (Cited 25 August 2014) 